\title{
Identification of the Domain Walls Configuration in the Ferroelastic Nanosize Material $\mathrm{La}_{0.95} \mathrm{Sr}_{0.05} \mathrm{Ga}_{0.9} \mathrm{Mg}_{0.1} \mathrm{O}_{3-x}$
}

\author{
D. Savytskit ${ }^{a, b}$, C. Paulmann ${ }^{c, d}$, U. Bismayer ${ }^{c}$ And M. Berkowski ${ }^{e}$ \\ ${ }^{a}$ Lviv Polytechnic National University, 12 Bandera St., 79013 Lviv, Ukraine \\ ${ }^{b}$ SRC "Carat", 202 Stryjska St., 79031 Lviv, Ukraine \\ ${ }^{c}$ Universität Hamburg, Grindelallee 48, D-20146 Hamburg, Germany \\ ${ }^{d}$ HASYLAB, DESY, Notkestr. 85, D-22603 Hamburg, Germany \\ ${ }^{e}$ Institute of Physics, al. Lotników 32/46, 02-668 Warsaw, Poland
}

\begin{abstract}
This paper deals with the identification of multidomain configuration in ferroelastic phases of $\mathrm{La}_{0.95} \mathrm{Sr}_{0.05} \mathrm{Ga}_{0.9} \mathrm{Mg}_{0.1} \mathrm{O}_{3-x}$ using polychromatic synchrotron X-ray radiation (Laue method). A nondestructive approach for the determination of domain misorientations, orientation of domain walls and their configuration in the nanosize ferroelastic domain structure was developed. The proposed approach can be used to study the nanosize ferroelastic domain structure in small crystals of submillimeter sizes at different external fields, including temperature. The ferroelastic domain structure in the orthorhombic as well as in the rhombohedral phases of $\mathrm{La}_{0.95} \mathrm{Sr}_{0.05} \mathrm{Ga}_{0.9} \mathrm{Mg}_{0.1} \mathrm{O}_{3-x}$ crystals has been identified. The intersection of walls leads to the formation of a chevron-like pattern. The observed reversibility of domain patterns during temperature cycles is probably caused by the interaction of domain boundaries with point defects, most likely oxygen vacancies.
\end{abstract}

PACS numbers: 61.72.Mm, 61.72.-y, 61.50.Ah

\section{Introduction}

Concerns about global warming due to increased emission of greenhouse gases and the depletion of fossil fuels have caused a renewed interest in alternative energy sources. Solid oxide fuel cells offer an environmentally advantageous alternative to classical engines generating electric power. One of the major technological problems with solid oxide fuel cells (SOFCs) is, however, to achieve high electrical conductivities. There is the tendency to develop SOFC electrolytes with higher conductivities at lower temperatures [1]. Literature search shows that all oxide electrolytes with high ionic conductivity show typical martensitic phase transitions which produce nanosize ferroelastic domain structures [2-6]. The high ionic conductivity of heavy-twinned electrolyte materials can be explained by different transport properties of domain walls and bulk areas of such compounds [7, 8].

The atomic size of twin walls and the nanosize dimensionality of domains made it difficult to get reliable experimental data about the influence of the interaction of point defects (oxygen vacancies) and ferroelastic domain walls with high ionic conductivity. The presence of ferroelastic domains often complicates or even does not allow us the detailed investigation of the physical properties in anisotropic ferroelastic phases. For example, due to different geometrical orientations of domains in a crystal it is difficult to perform the refinement of the crystal structure by single crystal methods [9].

Previous attempts to better understand the influence of the domain (twin) structure on the physical proper- ties were in general focused on computer simulation since experimental methods to identify domain wall configurations in samples with nanosize dimensionality were absent, except from high resolution transmission electron microscopy (HRTEM) [10-17]. The correlation between the ferroelastic domain structure with the point defect system as well as with physical properties requires knowledge about the configuration of domain walls in the ferroelastic phases.

HRTEM allows us to identify ferroelastic domain configurations in thin two-dimensional samples. Therefore we aim to develop a non-destructive method which allows us to identify the geometrical orientation of ferroelastic domains and the configuration of domain walls under the simultaneous influence of different types of external fields (thermal, mechanical, electrical, magnetic, etc.).

At the same time the "white" character of synchrotron radiation re-established the classical Laue method: it is now possible to record thousands of diffraction spots from crystalline samples in a short time using charge-coupled device (CCD) area detectors. For example, the divergence of the majority white beams at different synchrotron sources is smaller than a few milliradians and the space discriminatory limit $(20-60 \mu \mathrm{m}$ per pixel) of modern CCD-detectors allows us to fix the orientation contrast of the domain structure at a value of a few angle minutes. On the other side, the high intensity of synchrotron stations (more than 5 times higher than standard X-ray tubes) and the high sensitivity of CCD-detectors (40 electrons per a photon with $\lambda=1.54056 \AA$ ) 
allows us to reduce the Laue pattern exposition time to $0.1 \mathrm{~s}$. This tool can therefore be used for the characterization (determination of twin laws, misorientation of domains and domain wall orientations) of the twin structure, both in small crystals of submillimeter sizes [18, 19] and very small areas (few $\mu \mathrm{m}$ ) of larger samples (white beam X-ray microdiffraction) [20-22]. It can be used for in situ measurements under a variety of different experimental conditions, for studies of the reorientation of twin walls at phase transitions in particular. The advantage of this method over electron microscopy is that it is non-destructive, does not require complicated pre-experimental preparation of samples and allows us to use sample volumes up to several hundred $\mu \mathrm{m}$ size. Since an area detector collects data from all parts of the reciprocal space, the analysis of twinned crystals has become more common.

Three different theoretical approaches were used to analyze the parameters of the bidomain configuration of the $\mathrm{La}_{0.95} \mathrm{Sr}_{0.05} \mathrm{Ga}_{0.9} \mathrm{Mg}_{0.1} \mathrm{O}_{3-x}$ (LSGM05) twin structure [23]. For the determination of multidomain configurations in the investigated phases of LSGM05 we used the method which is based on transformation matrices [24].

The aim of this work is to identify all configurations of nanoscale domains in small $\mathrm{La}_{0.95} \mathrm{Sr}_{0.05} \mathrm{Ga}_{0.9} \mathrm{Mg}_{0.1} \mathrm{O}_{2.925}$ (LSGM05) ferroelastic crystals using the white beam $\mathrm{X}$-ray diffraction method.

\section{Sample preparation}

$\mathrm{La}_{1-x} \mathrm{Sr}_{x} \mathrm{Ga}_{1-2 x} \mathrm{Mg}_{2 x} \mathrm{O}_{3-\delta}$ solid solution crystals were grown using the Czochralski method. During the growth of the gallate compounds in argon atmosphere, the concentration of gallium oxide decreases with time because of the thermal dissociation of $\mathrm{Ga}_{2} \mathrm{O}_{3}$ and evaporation of the reaction products. Therefore, oxygen was introduced into the atmosphere in order to prevent the dissociation of the components during the growth. The best quality single crystal (Fig. 1) of $\mathrm{La}_{1-x} \mathrm{Sr}_{x} \mathrm{Ga}_{1-2 x} \mathrm{Mg}_{2 x} \mathrm{O}_{3-\delta}$ $(x=0.05), 35 \mathrm{~mm}$ long and $20 \mathrm{~mm}$ in diameter, was selected to study the twin structure. This crystal was grown from the stochiometric melt in argon/1\% oxygen atmosphere with a pulling rate of $1.2-2.5 \mathrm{~mm} / \mathrm{h}$. Chemical analysis confirmed that the cation composition of the crystal is identical to the initial concentration of its components [25]. The oxygen content was calculated based on charge neutrality.

In order to investigate the homogeneity of the received $\mathrm{La}_{0.95} \mathrm{Sr}_{0.05} \mathrm{Ga}_{0.9} \mathrm{Mg}_{0.1} \mathrm{O}_{3-\delta}$ (LSGM05) crystal microprobe analysis was performed. Measurement of the apparent concentrations of $\mathrm{La}, \mathrm{Ga}, \mathrm{Sr}$ and $\mathrm{Mg}$ was performed with a JEOL electron probe combined with a spectrum analyzer. Standards were $\mathrm{MgO}$, $\mathrm{SrTiO}_{3}, \mathrm{Nd}_{3} \mathrm{Ga}_{5} \mathrm{O}_{12}$ and a La-containing glass. Sample background and peak count times were $20 \mathrm{~s}$ with a beam current of $20 \mathrm{nA}$ and an acceleration voltage of $15 \mathrm{kV}$. The analysis showed good homogeneity of the $\mathrm{La}_{0.95} \mathrm{Sr}_{0.05} \mathrm{Ga}_{0.9} \mathrm{Mg}_{0.1} \mathrm{O}_{3-\delta}$ crystal. The scanned beam

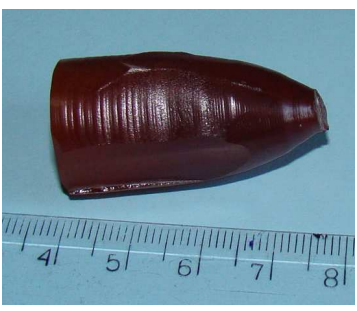

Fig. 1. $\mathrm{La}_{0.95} \mathrm{Sr}_{0.05} \mathrm{Ga}_{0.9} \mathrm{Mg}_{0.1} \mathrm{O}_{2.925}$ perovskite-type crystal grown by the Czochralski method.

images of $\mathrm{La} L_{\alpha}$, Ga $K_{\alpha}$, $\mathrm{Sr} L_{\alpha}$ and $\mathrm{Mg} K_{\alpha}$ from the $\mathrm{La}_{0.95} \mathrm{Sr}_{0.05} \mathrm{Ga}_{0.9} \mathrm{Mg}_{0.1} \mathrm{O}_{3-\delta}$ crystal are shown in Fig. 2.

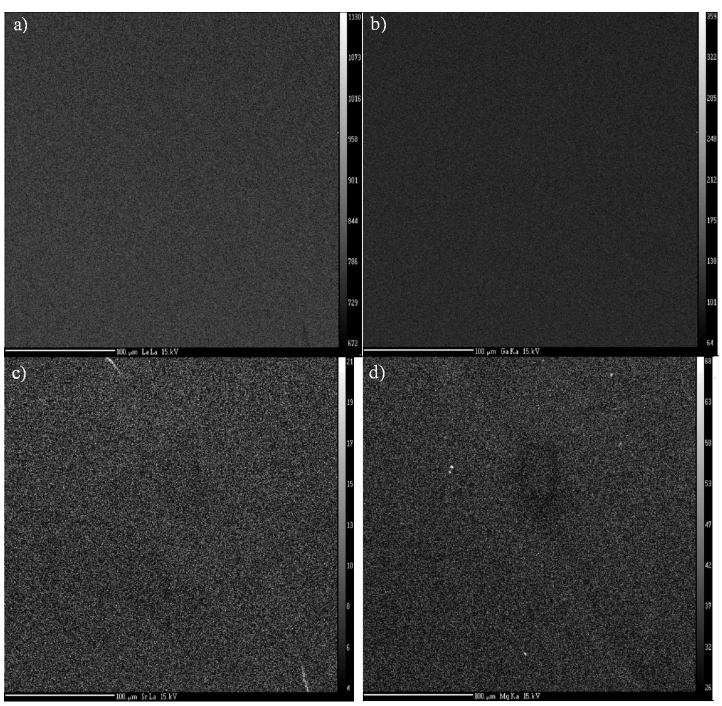

Fig. 2. Scanned beam images $300 \times 300 \mu \mathrm{m}$ of La $L_{\alpha}$ (a), Ga $K_{\alpha}$ (b), Sr $L_{\alpha}$ (c) and $\mathrm{Mg} K_{\alpha}$ (d) X-ray intensity from $\mathrm{La}_{0.95} \mathrm{Sr}_{0.05} \mathrm{Ga}_{0.9} \mathrm{Mg}_{0.1} \mathrm{O}_{3-\delta}$ crystal.

A crystal sample of $\mathrm{La}_{1-x} \mathrm{Sr}_{x} \mathrm{Ga}_{1-2 x} \mathrm{Mg}_{2 x} \mathrm{O}_{3-\delta}(x=$ $0.05)$ with $0.2 \times 0.2 \times 0.2 \mathrm{~mm}^{3}$ dimensions was selected using an optical microscope cut from a crystal boule. The sample was placed into a quartz capillary of $0.4 \mathrm{~mm}$ diameter and fixed using quartz wool.

\section{Measurements and processing data}

The Laue method was used to study in detail the twinning of the $\mathrm{La}_{0.95} \mathrm{Sr}_{0.05} \mathrm{Ga}_{0.9} \mathrm{Mg}_{0.1} \mathrm{O}_{2.925}$ perovskite-type crystal (LSGM05). Experiments were carried out at the F1 beamline at HASYLAB (DESY, Hamburg) using white X-ray synchrotron radiation. The used equipment includes a Kappa-diffractometer and a BRUKER CCD detector with a $6.25 \times 6.25 \mathrm{~cm}$ CDD matrix $(1024 \times 1024$ pixel resolution). The energy spectrum of the synchrotron beam was 5-60 keV. The CCD detector was positioned at $2 \theta=90^{\circ}$ to avoid exposure to the direct synchrotron beam.

The sample-to-detector distance was changed from $58 \mathrm{~mm}$ to $358 \mathrm{~mm}$ depending on the experimental task. 
A longer distance provides a diffraction picture with few multiplets and higher spatial resolution of reflections from each domain state. On the other hand, a shorter distance allows us to observe a larger number of multiplets which are necessary to index and determine the orientation matrix. Angular scanning of the sample was carried out around two axes using the conditions, $\omega=0^{\circ}$, $\phi$ axis $=0^{\circ}-180^{\circ}$ with $15^{\circ}$ step, $\chi$ axis $=0^{\circ}-90^{\circ}$ with $15^{\circ}$ step width. A Laue diffraction pattern with the biggest spacial splitting of reflections from different twin domains was selected for initial indexing. The temperature of the sample was changed using a nitrogen gas stream heated to the proper temperature. A thermocouple was used to preliminarily calibrate the sample temperature.

OrientExpress V3.3 freeware available via the internet [26] was used to index the Laue diffraction patterns. Input data include parameters of the crystal lattice, sample-to-detector distance, wave band of synchrotron radiation, dimensions of the matrix and angular position of the detector, and reflection coordinates. We developed an algorithm to specify and precisely measure the sample-detector distance $(d)$ and to determine coordinates of the projection of the sample position in the detection plane of the CCD detector. The algorithm implies the analysis of two Laue diffraction patterns detected at two different distances $d_{\mathrm{F} 1}$ (for example, $d_{1}=100 \mathrm{~mm}$ and $d_{2}=50 \mathrm{~mm}$ ) but at the same angular position of the sample. Distances $d_{1}$ and $d_{2}$ are the distances from the sample to the beryllium window of the CCD chamber and not to the detection plane of the CCD detector. Therefore, corrections are needed. The algorithm also implies that the coordinates of the same Bragg reflections in pixel coordinates of the CCD detector are determined for two different Laue diffraction patterns.

The measured Laue diffraction patterns contain Bragg reflections and diffuse scattering. Additionally, there is a rather intensive background because the white synchrotron beam is scattered from the capillary, quartz-wool and the sample surface. Since the background was dome-shaped at short sample-to-detector distances (Fig. 3), a 4th order polynomial was used for its approximation in both, $x$-columns and $y$-rows. A final background value at each $(x, y)$ point was the average of two values derived through approximation in $x$ column and $y$ row. The points, where the derivative exceeds a specified threshold $(p)$ were removed from the array used for calculating the background. In other words, we removed from the neighborhood of the Bragg reflections and diffuse scattering those high intensity points which would considerably "distort" the background lines (Figs. 3 and 4). The threshold $p$ was around 5-20 units. Smaller values did not provide a better approximation of the background because too many points would be removed from the array due to the white noise modulation of the Laue diffraction pattern. It should be noted that the optimum $p$ value depends on the sample-detector distance because the shape and intensity of the background are specific to the distance.
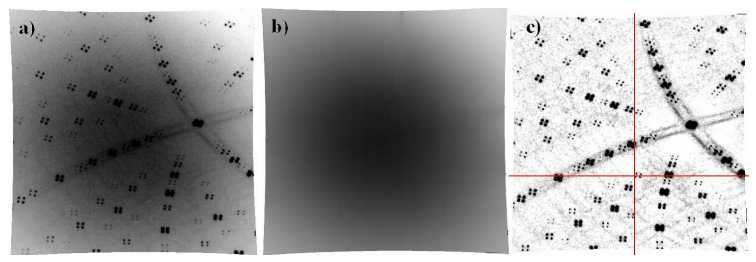

Fig. 3. Laue diffraction pattern recorded at $58 \mathrm{~mm}$ : (a) before removing (b) the background, (c) after removing the background. Lines indicate $X$ and $Y$ profiles shown in Fig. 4.

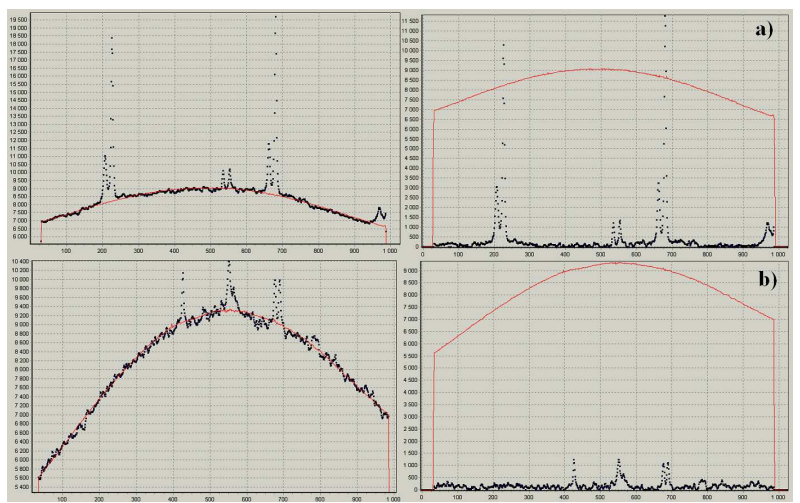

Fig. 4. $X(y=690 \mathrm{px})$ and $Y(x=536 \mathrm{px})$ profiles of the Laue diffraction pattern recorded at $58 \mathrm{~mm}$ : (a) before removing the background, (b) after removing the background. Solid line indicates the calculated background.

After subtracting the background a Pearson VII two-variable function was used to approximate the profile of the Bragg reflections

$$
\begin{gathered}
Z(x, y)=I_{0}\left[1+\left(\frac{2\left(x-x_{0}\right) \sqrt{2^{1 / 7}-1}}{w}\right)^{2}\right]^{-7} \\
\times\left[1+\left(\frac{2\left(y-y_{0}\right) \sqrt{2^{1 / 7}-1}}{w}\right)^{2}\right]^{-7}
\end{gathered}
$$

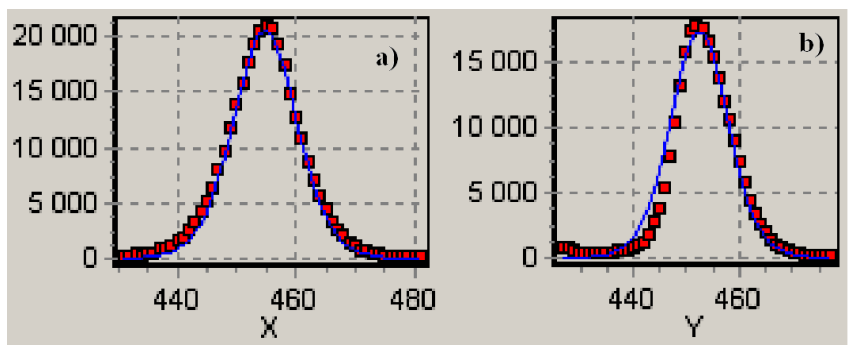

Fig. 5. Profiles of the reflection detected at distance $358 \mathrm{~mm}$ : (a) $X$-profile, (b) $Y$-profile. Parameters of Pearson VII approximation: $I_{0}=20579$ a.u., $x_{0}=$ $454.8 \mathrm{px}, y_{0}=452.4 \mathrm{px}, w=12.6 \mathrm{px}$. 
where $I_{0}$ is intensity of reflection, $x_{0}$ and $y_{0}$ are coordinates of reflection center, $w$ is full width at half maximum. We used different $w$ values for $x$ and $y$ variables. However, the analysis of the data provide close $w_{x}$ and $w_{y}$ values, that allows to use the same $w$ value for both $x$ and $y$ arguments to ensure sufficient approximation of the profiles (Fig. 5).
Having determined the coordinates of the projection of the sample position in the recording plane of the CCD detector and the coordinates of Bragg reflection centers in the CCD detector setting, we calculated the reflection coordinates in the laboratory system. Such results were the input data for indexing the Laue diffraction patterns using OrientExpress V3.3 software.

Solutions obtained by indexing reflections from the orientation state TO1 and their statistics.

TABLE I

\begin{tabular}{|c|c|c|c|c|c|c|c|c|c|c|c|c|c|c|c|c|c|c|c|c|c|c|c|c|}
\hline \multirow{2}{*}{$\begin{array}{l}\text { Reflection's } \\
\text { number }\end{array}$} & \multicolumn{4}{|c|}{ 1st solution } & \multicolumn{4}{|c|}{ 2nd solution } & \multicolumn{4}{|c|}{ 3rd solution } & \multicolumn{4}{|c|}{ 4th solution } & \multicolumn{4}{|c|}{5 th solution } & \multicolumn{4}{|c|}{6 th solution } \\
\hline & $h$ & $k$ & $l$ & $\begin{array}{c}\text { Error } \\
{[\mathrm{cm}]}\end{array}$ & $h$ & $k$ & $l$ & $\begin{array}{c}\text { Error } \\
{[\mathrm{cm}]}\end{array}$ & $h$ & $k$ & $l$ & $\begin{array}{c}\text { Error } \\
{[\mathrm{cm}]}\end{array}$ & $h$ & $k$ & $l$ & $\begin{array}{c}\text { Error } \\
{[\mathrm{cm}]}\end{array}$ & $h$ & $k$ & $l$ & $\begin{array}{c}\text { Error } \\
{[\mathrm{cm}]}\end{array}$ & $h$ & $k$ & $l$ & $\begin{array}{c}\text { Error } \\
{[\mathrm{cm}]}\end{array}$ \\
\hline 1 & 1 & 4 & 2 & 0.004 & 2 & 4 & 1 & 0.005 & 7 & 2 & 1 & 0.002 & 5 & 6 & 3 & 0.001 & 3 & 6 & 5 & 0.004 & 1 & 2 & 7 & 0.033 \\
\hline 2 & 1 & 4 & 1 & 0.005 & 1 & 4 & 1 & 0.016 & 3 & 0 & 1 & 0.009 & 1 & 1 & 1 & 0.001 & 1 & 1 & 1 & 0.015 & 1 & 0 & 3 & 0.029 \\
\hline 3 & 3 & 6 & 1 & 0.007 & 1 & 6 & 3 & 0.017 & 5 & -2 & 1 & 0.012 & 1 & 2 & 2 & 0.004 & 2 & 2 & 1 & 0.019 & 1 & -2 & 5 & 0.049 \\
\hline 4 & 3 & 10 & 9 & 0.007 & 9 & 10 & 3 & 0.013 & 11 & 6 & -1 & 0.011 & 4 & 6 & 1 & 0.002 & 1 & 6 & 4 & 0.019 & -1 & 6 & 11 & 0.056 \\
\hline 5 & 1 & 4 & 3 & 0.003 & 3 & 4 & 1 & 0.007 & 2 & 1 & 0 & 0.007 & 3 & 4 & 1 & 0.002 & 1 & 4 & 3 & 0.012 & 0 & 1 & 2 & 0.049 \\
\hline 6 & 3 & 14 & 5 & 0.005 & 5 & 14 & 3 & 0.014 & 11 & 2 & 3 & 0.009 & 4 & 4 & 3 & 0.002 & 3 & 4 & 4 & 0.013 & 3 & 2 & 11 & 0.027 \\
\hline 7 & 2 & 4 & 1 & 0.002 & 1 & 4 & 2 & 0.015 & 7 & -2 & 1 & 0.011 & 3 & 6 & 5 & 0.002 & 5 & 6 & 3 & 0.016 & 1 & -2 & 7 & 0.044 \\
\hline 8 & 5 & 10 & 3 & 0.003 & 3 & 10 & 5 & 0.016 & 9 & -2 & 1 & 0.012 & 2 & 4 & 3 & 0.003 & 3 & 4 & 2 & 0.017 & & & & \\
\hline 9 & 3 & 6 & 2 & 0.002 & 2 & 6 & 3 & 0.014 & 11 & -2 & 3 & 0.005 & 5 & 10 & 7 & 0.001 & 7 & 10 & 5 & 0.015 & & & & \\
\hline 10 & 5 & 14 & 3 & 0.004 & 3 & 14 & 5 & 0.011 & 13 & -2 & 3 & 0.003 & 3 & 4 & 4 & 0 & 4 & 4 & 3 & 0.005 & & & & \\
\hline 11 & 3 & 8 & 2 & 0.002 & 2 & 8 & 3 & 0.009 & 4 & 0 & 1 & 0.005 & 7 & 10 & 9 & 0.001 & 9 & 10 & 7 & 0.004 & 1 & 0 & 4 & 0.03 \\
\hline 12 & 3 & 10 & 3 & 0.004 & 3 & 10 & 3 & 0.01 & 5 & 0 & 1 & 0.004 & 5 & 6 & 5 & 0.001 & 5 & 6 & 5 & 0.009 & 1 & 0 & 5 & 0.03 \\
\hline 13 & 1 & 3 & 1 & 0.004 & 1 & 3 & 1 & 0.007 & 6 & 0 & 1 & 0.005 & 3 & 4 & 3 & 0 & 3 & 4 & 3 & 0.005 & 1 & 0 & 6 & 0.032 \\
\hline 14 & 5 & 14 & 5 & 0.005 & 5 & 14 & 5 & 0.008 & 13 & 2 & 3 & 0.005 & 7 & 10 & 7 & 0.001 & 7 & 10 & 7 & 0.006 & 3 & 2 & 13 & 0.028 \\
\hline 15 & 2 & 8 & 3 & 0.004 & 3 & 8 & 2 & 0.009 & 5 & 2 & 1 & 0.008 & 9 & 10 & 7 & 0 & 7 & 10 & 9 & 0.009 & 1 & 2 & 5 & 0.034 \\
\hline 16 & 1 & 6 & 3 & 0.004 & 3 & 6 & 1 & 0.01 & 6 & 2 & 1 & 0.005 & 2 & 2 & 1 & 0.001 & 1 & 2 & 2 & 0.005 & 1 & 2 & 6 & 0.034 \\
\hline 17 & 3 & 14 & 7 & 0.003 & 7 & 14 & 3 & 0.008 & 9 & 2 & 1 & 0.002 & 9 & 10 & 5 & 0.002 & 5 & 10 & 9 & 0.006 & 1 & 2 & 9 & 0.033 \\
\hline 18 & 3 & 10 & 5 & 0.005 & 5 & 10 & 3 & 0.003 & 11 & 2 & 1 & 0.003 & 3 & 4 & 2 & 0.002 & 2 & 4 & 3 & 0.003 & 1 & 2 & 11 & 0.033 \\
\hline 19 & 2 & 6 & 3 & 0.005 & 3 & 6 & 2 & 0.003 & 11 & 6 & 1 & 0.009 & 7 & 10 & 5 & 0.001 & 5 & 10 & 7 & 0.002 & 1 & 6 & 11 & 0.05 \\
\hline 20 & 1 & 6 & 4 & 0.006 & 4 & 6 & 1 & 0.009 & 5 & 2 & 0 & 0.004 & 9 & 10 & 3 & 0.002 & 3 & 10 & 9 & 0.011 & 0 & 2 & 5 & 0.044 \\
\hline 21 & 3 & 10 & 7 & 0.002 & 7 & 10 & 3 & 0.004 & 3 & 1 & 0 & 0.004 & 7 & 10 & 3 & 0.002 & 3 & 10 & 7 & 0.006 & 0 & 1 & 3 & 0.041 \\
\hline 22 & 1 & 3 & 2 & 0.003 & 2 & 3 & 1 & 0.004 & 11 & 2 & -1 & 0.014 & 2 & 3 & 1 & 0.002 & 1 & 3 & 2 & 0.004 & -1 & 2 & 11 & 0.042 \\
\hline 23 & 5 & 10 & 7 & 0.006 & 7 & 10 & 5 & 0.014 & 6 & -2 & 1 & 0.011 & 3 & 6 & 2 & 0.002 & 2 & 6 & 3 & 0.012 & 1 & -2 & 6 & 0.044 \\
\hline 24 & 7 & 14 & 3 & 0.004 & 3 & 14 & 7 & 0.015 & 5 & 0 & 2 & 0.012 & 5 & 10 & 9 & 0.002 & 9 & 10 & 5 & 0.017 & 2 & 0 & 5 & 0.033 \\
\hline 25 & 3 & 14 & 3 & 0.006 & 3 & 14 & 3 & 0.024 & 13 & 6 & 1 & 0.004 & 7 & 6 & 7 & 0.003 & 7 & 6 & 7 & 0.023 & & & & \\
\hline 26 & 3 & 14 & 9 & 0.002 & 9 & 14 & 3 & 0.006 & 7 & 0 & 1 & 0.006 & 5 & 6 & 2 & 0.003 & 2 & 6 & 5 & 0.006 & 1 & 0 & 7 & 0.034 \\
\hline 27 & 3 & 8 & 3 & 0.006 & 3 & 8 & 3 & 0.008 & 13 & 2 & 1 & 0.005 & 2 & 3 & 2 & 0.002 & 2 & 3 & 2 & 0.006 & 1 & 2 & 13 & 0.034 \\
\hline 28 & 5 & 14 & 7 & 0.006 & 7 & 14 & 5 & 0.005 & 13 & 2 & 5 & 0.008 & 4 & 6 & 3 & 0.001 & 3 & 6 & 4 & 0.004 & 5 & 2 & 13 & 0.029 \\
\hline 30 & 1 & 2 & 1 & 0.005 & 1 & 2 & 1 & 0.015 & 1 & 0 & 0 & 0.012 & 1 & 2 & 1 & 0.002 & 1 & 2 & 1 & 0.015 & 0 & 0 & 1 & 0.038 \\
\hline $\begin{array}{c}\text { Mean } \\
\text { deviation }\end{array}$ & \multicolumn{4}{|c|}{0.0043} & \multicolumn{4}{|c|}{0.0103} & \multicolumn{4}{|c|}{0.0071} & \multicolumn{4}{|c|}{0.0017} & \multicolumn{4}{|c|}{0.0099} & \multicolumn{4}{|c|}{0.0372} \\
\hline $\begin{array}{c}\text { Sample-CCD } \\
\text { dist. }[\mathrm{mm}]\end{array}$ & \multicolumn{4}{|c|}{58.0} & \multicolumn{4}{|c|}{58.0} & \multicolumn{4}{|c|}{58.2} & \multicolumn{4}{|c|}{58.0} & \multicolumn{4}{|c|}{57.9} & \multicolumn{4}{|c|}{57.3} \\
\hline
\end{tabular}

\section{Indexing Laue diffraction patterns}

\subsection{Orthorhombic phase}

Figure 6 a shows a section of a Laue diffraction pattern of a LSGM05 crystal detected at room temperature with a crystal-detector distance of $d=58 \mathrm{~mm}$. The Laue pattern shows multiplets splitted into 4 reflections, each one generated by the Bragg reflections from its corresponding geometrical orientation state. The symbols TO1, TO2, TO3 and TO4 (Fig. 6a) indicate different ferroelastic orientation states with corresponding reflections. Positions of reflections (up to 30 in total) were used to refine the orientation matrix and sample-to-detector distance. The orthorhombic cell with parameters $a=5.499 \AA$, $b=7.794 \AA, c=5.538 \AA[25]$ were used for indexing the Laue diffraction patterns. To start indexing, the first 
six multiplets were selected at intersections of crystallographic zones. These reflections had small Miller indices which ensures that OrientExpress-assisted indexing leads to correct solutions.

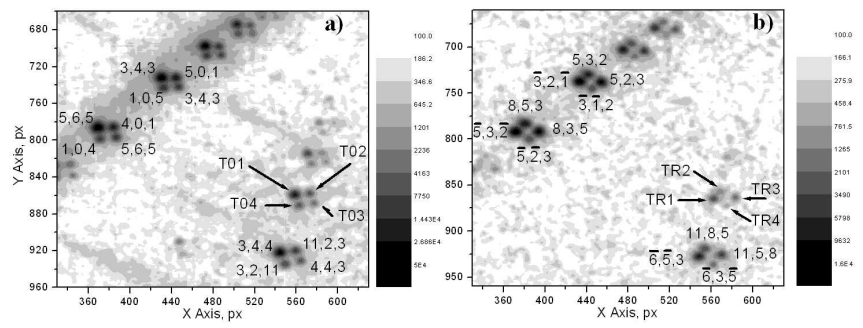

Fig. 6. Sections of Laue diffraction patterns of LSGM05 detected at (a) $300 \mathrm{~K}$ and (b) $753 \mathrm{~K}(d=$ $\left.58 \mathrm{~mm}, \varphi=87^{\circ}, \psi=15^{\circ}\right)$. Reflections are indexed according to the best solution determined for each of the four domain states.

After the initial indexing of 6 reflections of the TO1 orientation state $\left(0.2^{\circ}\right.$ error $)$ we obtained about 20 different solutions. Only 6 solutions coincide with the experimental Laue diffraction pattern and possess relatively small differences between the calculated and experimental reflection positions. These 6 solutions correspond to 6 allowed domain states in the ferroelastic orthorhombic phase of LSGM05 [23]. Further refinement of these solutions using 30 reflections is given in Table I. The minimum mean deviation (several times smaller than in the other five cases) is ensured by the 4 th solution. In this case the sample-to-CCD detector distance remains the same and is $58.0 \mathrm{~mm}$. The results show the indices of the 4th solution and its corresponding orientation matrix (determined by OrientExpress and shown in Table II). OrientExpress software allows for the estimation of an angular error for each solution. This error was below $0.02^{\circ}$ for the best (4th) solution.

Results of the Bragg reflections for the remaining 3 domain states (TO2, TO3, and TO4) are given in Table II. Indexing of reflections for each domain state yields the same 6 solutions as for the state TO1. However, consideration of more reflections has shown that the 3rd solution is the best for the state TO2, the 5th for TO3, and the 6 th for TO4 (bold in Table II). The mean deviation provided by these best solutions is several times smaller than in other cases, with a sample-to-detector distance remaining $58.0 \mathrm{~mm}$.

TABLE II

Solutions obtained by indexing reflections from the orientation states TO1-TO4 and their statistics.

\begin{tabular}{c|c|c|c|c|c|c|c|c}
\hline \hline Domain & \multicolumn{2}{|c|}{ TO1 } & \multicolumn{2}{c|}{ TO2 } & \multicolumn{2}{c|}{ TO3 } & \multicolumn{2}{c}{ TO4 } \\
\hline Solution & $\begin{array}{c}\text { Mean dev. } \\
{[\mathrm{mm}]}\end{array}$ & $\begin{array}{c}\text { Sample-CCD } \\
\text { dist. [mm] }\end{array}$ & $\begin{array}{c}\text { Mean dev. } \\
{[\mathrm{mm}]}\end{array}$ & $\begin{array}{c}\text { Sample-CCD } \\
\text { dist. }[\mathrm{mm}]\end{array}$ & $\begin{array}{c}\text { Mean dev. } \\
{[\mathrm{mm}]}\end{array}$ & $\begin{array}{c}\text { Sample-CCD } \\
\text { dist. [mm] }\end{array}$ & $\begin{array}{c}\text { Mean dev. } \\
{[\mathrm{mm}]}\end{array}$ & $\begin{array}{c}\text { Sample-CCD } \\
\text { dist. [mm] }\end{array}$ \\
\hline 1-solution & 0.043 & 58.0 & 0.076 & 57.8 & 0.096 & 58.0 & 0.050 & 58.4 \\
2-solution & 0.103 & 58.0 & 0.070 & 57.8 & 0.056 & 58.0 & 0.067 & 58.4 \\
3-solution & 0.071 & 58.2 & $\mathbf{0 . 0 1 7}$ & $\mathbf{5 8 . 0}$ & 0.096 & 58.2 & 0.066 & 58.6 \\
4-solution & $\mathbf{0 . 0 1 7}$ & $\mathbf{5 8 . 0}$ & 0.091 & 57.9 & 0.102 & 58.2 & 0.087 & 58.5 \\
5-solution & 0.099 & 57.9 & 0.087 & 57.7 & $\mathbf{0 . 0 2 2}$ & $\mathbf{5 8 . 0}$ & 0.060 & 58.3 \\
6-solution & 0.372 & 57.3 & 0.140 & 57.5 & 0.069 & 57.9 & $\mathbf{0 . 0 2 2}$ & $\mathbf{5 8 . 0}$
\end{tabular}

It should be mentioned that reflections from each domain are indexed independently. In the orthorhombic symmetry, the Laue diffraction patterns can be indexed according to 8 different crystallographic equivalent settings with the same indices, they differ only by sign.

\subsection{Trigonal phase}

The Laue diffraction patterns show that heating of the sample above $720 \mathrm{~K}$ to the trigonal phase causes the multiplets to split into 4 reflections. The symbols TR1, TR2, TR3 and TR4 (Fig. 6b) indicate the corresponding orientation states. All Laue diffraction patterns were separately indexed using the reflections of each of the 4 domain states. Positions of reflections (up to 29 in total) were used to refine the orientation matrix $\boldsymbol{M}_{T j}$ and sample-to-detector distance. Powder diffraction shows that the trigonal cell with space group $R 3 c$ and parameters $a=5.527 \AA ; \alpha=60.33^{\circ}$ is characteristic for the
LSGM05 crystal at $753 \mathrm{~K}[25]$. Those parameters were used for indexing the Laue diffraction patterns.

After initial indexing of 6 reflections of the orientation state TR $1\left(0.2^{\circ}\right.$ error $)$ we have obtained 4 different solutions, which visually coincide with the experimental Laue diffraction pattern. Further refinement of these solutions using 30 reflections is given in Table III. The minimum mean deviation for the state TR1 results for the 3rd solution. In this case, the sample-to-detector distance remains the same and is $58.0 \mathrm{~mm}$. The results allow to index the TR1 domain reflections using indices of the 3rd solution. This solution was obtained with an angular error below $0.03^{\circ}$.

Results of the Bragg reflections indexed for the other 3 domain states (TR2, TR3 and TR4) are given in Table IV. Indexing of reflections for each domain state yields the same 4 solutions as for the state TR1. However, consideration of more reflections (up to 29) has shown 
that the 1st solution is the best for the state TR2, the 4th for TR3, and the 2nd for TR4 (marked by bold in Table IV). Similarly to the state TR1, the mean deviation provided by such best solutions is several times smaller than in other cases.

TABLE III

Results of indexing reflections from the state TR1 and their statistics.

\begin{tabular}{|c|c|c|c|c|c|c|c|c|c|c|c|c|c|c|c|c|}
\hline \multirow{2}{*}{$\begin{array}{c}\text { Reflection's } \\
\text { number }\end{array}$} & \multicolumn{4}{|c|}{ 1st solution } & \multicolumn{4}{|c|}{ 2nd solution } & \multicolumn{4}{|c|}{ 3rd solution } & \multicolumn{4}{|c|}{ 4th solution } \\
\hline & $h$ & $k$ & $l$ & $\begin{array}{c}\text { Error } \\
{[\mathrm{cm}]}\end{array}$ & $h$ & $k$ & $l$ & $\begin{array}{c}\text { Error } \\
{[\mathrm{cm}]}\end{array}$ & $h$ & $k$ & $l$ & $\begin{array}{c}\text { Error } \\
{[\mathrm{cm}]}\end{array}$ & $h$ & $k$ & $l$ & $\begin{array}{c}\text { Error } \\
{[\mathrm{cm}]}\end{array}$ \\
\hline 1 & 2 & 1 & 1 & 0.021 & -1 & 0 & 1 & 0.017 & -1 & 1 & 0 & 0.005 & 2 & 1 & 1 & 0.022 \\
\hline 2 & 7 & 5 & 4 & 0.019 & -3 & -1 & 4 & 0.013 & -5 & 2 & -1 & 0.002 & 7 & 2 & 3 & 0.012 \\
\hline 3 & 3 & 2 & 1 & 0.015 & -2 & -1 & 1 & 0.019 & -2 & 1 & -1 & 0.002 & 3 & 1 & 2 & 0.023 \\
\hline 4 & 5 & 2 & 1 & 0.025 & -4 & -1 & 1 & 0.008 & -2 & 3 & -1 & 0.001 & 5 & 3 & 4 & 0.018 \\
\hline 5 & 7 & 3 & 2 & 0.021 & -5 & -1 & 2 & 0.01 & -3 & 4 & -1 & 0.002 & 7 & 4 & 5 & 0.017 \\
\hline 6 & 8 & 5 & 3 & 0.012 & -5 & -2 & 3 & 0.009 & -5 & 3 & -2 & 0.001 & 8 & 3 & 5 & 0.013 \\
\hline 7 & 9 & 4 & 3 & 0.022 & -6 & -1 & 3 & 0.013 & -4 & 5 & -1 & 0.003 & 9 & 5 & 6 & 0.018 \\
\hline 8 & 11 & 6 & 3 & 0.016 & -8 & -3 & 3 & 0.01 & -6 & 5 & -3 & 0.002 & 11 & 5 & 8 & 0.01 \\
\hline 9 & 5 & 3 & 2 & 0.012 & -3 & -1 & 2 & 0.004 & -3 & 2 & -1 & 0 & 5 & 2 & 3 & 0.007 \\
\hline 10 & 12 & 7 & 5 & 0.013 & -7 & -2 & 5 & 0.002 & -7 & 5 & -2 & 0.001 & 12 & 5 & 7 & 0.004 \\
\hline 11 & 11 & 8 & 5 & 0.021 & -6 & -3 & 5 & 0.016 & -8 & 3 & -3 & 0.002 & 11 & 3 & 6 & 0.023 \\
\hline 12 & 5 & 4 & 3 & 0.028 & -2 & -1 & 3 & 0.017 & -4 & 1 & -1 & 0.003 & 5 & 1 & 2 & 0.017 \\
\hline 13 & 12 & 9 & 7 & 0.023 & -5 & -2 & 7 & 0.014 & -9 & 3 & -2 & 0.001 & 12 & 3 & 5 & 0.015 \\
\hline 14 & 9 & 6 & 5 & 0.015 & -4 & -1 & 5 & 0.01 & -6 & 3 & -1 & 0.001 & 9 & 3 & 4 & 0.008 \\
\hline 15 & 11 & 7 & 6 & 0.013 & -5 & -1 & 6 & 0.008 & -7 & 4 & -1 & 0.001 & 11 & 4 & 5 & 0.007 \\
\hline 16 & 11 & 9 & 8 & 0.034 & -3 & -1 & 8 & 0.025 & -9 & 2 & -1 & 0.002 & 11 & 2 & 3 & 0.011 \\
\hline 17 & 4 & 3 & 3 & 0.021 & -1 & 0 & 3 & 0.027 & -3 & 1 & 0 & 0.002 & 4 & 1 & 1 & 0.005 \\
\hline 18 & 10 & 7 & 7 & 0.017 & -3 & 0 & 7 & 0.02 & -7 & 3 & 0 & 0.001 & 10 & 3 & 3 & 0.009 \\
\hline 19 & 3 & 2 & 2 & 0.015 & -1 & 0 & 2 & 0.018 & -2 & 1 & 0 & 0 & 3 & 1 & 1 & 0.009 \\
\hline 20 & 11 & 8 & 9 & 0.021 & -2 & 1 & 9 & 0.033 & -8 & 3 & 1 & 0.002 & 11 & 3 & 2 & 0.012 \\
\hline 21 & 5 & 3 & 4 & 0.022 & -1 & 1 & 4 & 0.034 & -3 & 2 & 1 & 0.003 & 5 & 2 & 1 & 0.027 \\
\hline 22 & 7 & 4 & 5 & 0.018 & -2 & 1 & 5 & 0.026 & -4 & 3 & 1 & 0.002 & 7 & 3 & 2 & 0.024 \\
\hline 23 & 9 & 5 & 6 & 0.019 & -3 & 1 & 6 & 0.023 & -5 & 4 & 1 & 0.001 & 9 & 4 & 3 & 0.024 \\
\hline 24 & 10 & 7 & 3 & 0.019 & -7 & -4 & 3 & 0.029 & -7 & 3 & -4 & 0.004 & 10 & 3 & 7 & 0.03 \\
\hline 25 & 13 & 9 & 6 & 0.016 & -7 & -3 & 6 & 0.012 & -9 & 4 & -3 & 0.002 & 13 & 4 & 7 & 0.017 \\
\hline 26 & 11 & 5 & 4 & 0.019 & -7 & -1 & 4 & 0.012 & -5 & 6 & -1 & 0.001 & 11 & 6 & 7 & 0.016 \\
\hline 27 & 11 & 6 & 7 & 0.017 & -4 & 1 & 7 & 0.023 & -6 & 5 & 1 & 0.002 & 11 & 5 & 4 & 0.023 \\
\hline 28 & 13 & 10 & 9 & 0.024 & -4 & -1 & 9 & 0.019 & -10 & 3 & -1 & 0.002 & 13 & 3 & 4 & 0.008 \\
\hline 29 & 12 & 7 & 9 & 0.023 & -3 & 2 & 9 & 0.029 & -7 & 5 & 2 & 0.003 & 12 & 5 & 3 & 0.029 \\
\hline $\begin{array}{c}\text { Mean } \\
\text { deviation }\end{array}$ & \multicolumn{4}{|c|}{0.01934} & \multicolumn{4}{|c|}{0.01724} & \multicolumn{4}{|c|}{0.00186} & \multicolumn{4}{|c|}{0.01579} \\
\hline $\begin{array}{l}\text { Sample-CCD } \\
\text { distance }[\mathrm{mm}]\end{array}$ & \multicolumn{4}{|c|}{58.2} & \multicolumn{4}{|c|}{58.0} & \multicolumn{4}{|c|}{58.0} & \multicolumn{4}{|c|}{58.0} \\
\hline
\end{tabular}

TABLE IV

Results of indexing reflections from the orientation states TR1-TR4 at $753 \mathrm{~K}$ and their statistics.

\begin{tabular}{c|c|c|c|c|c|c|c|c}
\hline \hline Domain & \multicolumn{2}{|c|}{ TR1 } & \multicolumn{2}{c|}{ TR2 } & \multicolumn{2}{|c|}{ TR3 } & TR4 \\
\hline Solution & $\begin{array}{c}\text { Mean dev. } \\
{[\mathrm{mm}]}\end{array}$ & $\begin{array}{c}\text { Sample-CCD } \\
\text { dist. [mm] }\end{array}$ & $\begin{array}{c}\text { Mean dev. } \\
{[\mathrm{mm}]}\end{array}$ & $\begin{array}{c}\text { Sample-CCD } \\
\text { dist. [mm] }\end{array}$ & $\begin{array}{c}\text { Mean dev. } \\
{[\mathrm{mm}]}\end{array}$ & $\begin{array}{c}\text { Sample-CCD } \\
\text { dist. [mm] }\end{array}$ & $\begin{array}{c}\text { Mean dev. } \\
{[\mathrm{mm}]}\end{array}$ & $\begin{array}{c}\text { Sample-CCD } \\
\text { dist. [mm] }\end{array}$ \\
\hline 1-solution & 0.193 & 58.2 & $\mathbf{0 . 0 6 0}$ & $\mathbf{5 8 . 0}$ & 0.048 & 58.1 & 0.065 & 58.3 \\
2-solution & 0.172 & 58.0 & 0.121 & 57.5 & 0.090 & 57.8 & $\mathbf{0 . 0 2 8}$ & $\mathbf{5 8 . 0}$ \\
3-solution & $\mathbf{0 . 0 1 9}$ & $\mathbf{5 8 . 0}$ & 0.149 & 57.6 & 0.150 & 58.0 & 0.110 & 57.9 \\
4-solution & 0.158 & 58.0 & 0.122 & 57.9 & $\mathbf{0 . 0 2 4}$ & $\mathbf{5 8 . 0}$ & 0.055 & 58.4
\end{tabular}

We used the OrientExpress software for indexing each domain separately. It should be mentioned that the row sequence in orientation matrices $\boldsymbol{M}_{T j}$ and the sign depend upon the setting used for indexing. The sequence of 
rows in calculated matrices $\boldsymbol{M}_{T j}$ can be changed through cyclic permutation using the basis vector equivalency of a rhombohedral cell. Centrosymmetrical Laue diffraction patterns allow us multiplying rows of $\boldsymbol{M}_{T j}$ by -1 .

\section{Identification of domain walls}

To avoid ambiguous identification of domain structures we used the "relative shifts" method, an algorithm which is shown in Fig. 7. The proposed method is based on the selection of one domain referred as "reference" domain, the determination of spot coordinates from this domain state on the Laue pattern and indexing selected reflections with subsequent determination of the orientation matrice $\boldsymbol{M}_{1}$ of the domain state relative to the laboratory coordinate system. In next step, using the transformation matrices $\boldsymbol{T}_{1 t}$ (see Table 1-3 in [23]), we determined the orientation matrices ${ }^{1} \boldsymbol{M}_{t}$ of all possible domain states according to Eq. (9) [23] and performed calculations with respect to the orientation matrix $\boldsymbol{M}_{1}$ of the reference domain. Using orientation matrices ${ }^{1} \boldsymbol{M}_{t}$, we simulated positions of the Bragg reflections of all possible domain states on the Laue diffraction pattern and compared the results with observed reflections. Based on symmetry laws (domain wall orientation) positions of simulated and experimental reflections were compared. Using this method, it was possible to analyze complex multidomain configurations of ferroelastic structures.

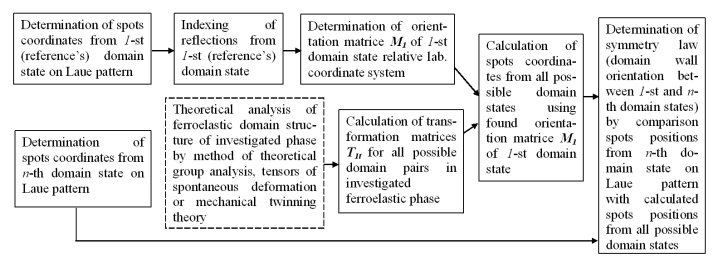

Fig. 7. An algorithm of the "relative shifts" method for identification of domain wall configurations in ferroelastic crystals.

\subsection{Trigonal phase}

Figure 8a shows an enlarged part of the Laue diffraction pattern obtained at a distance of $358 \mathrm{~mm}$. In addition to the observed Bragg reflections from four TR domains, the calculated positions of reflections from allowed domain states in this phase are marked by circles. The calculated positions of the Bragg reflections from domain states connected with "reference" domain TR1 by (011) and (121) mirror plane coincide with the positions of the (211) reflections from domains TR2 and TR3. Figure $8 \mathrm{a}$ shows that the calculated positions of reflections from all other potential domains are outside the region of the observed reflections including the reflection from domain TR4. This indicates that there are no stress-free walls between the pair of domain states TR1-TR4.

In a next step, using the transformation matrices $\boldsymbol{T}_{1 t}$ (Table 1 in [23]), we determined the orientation matrices

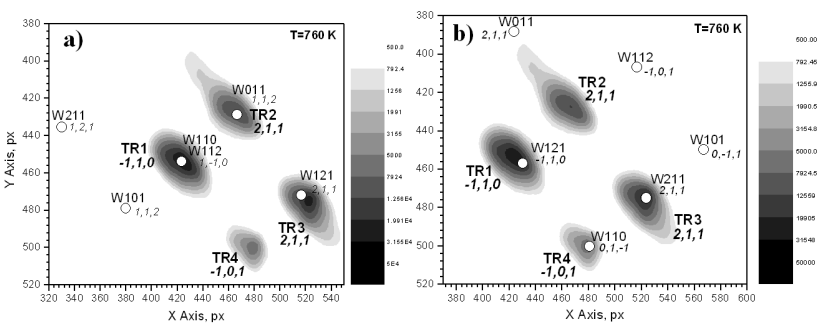

Fig. 8. Enlarged fragment of the Laue diffraction pattern observed at $358 \mathrm{~mm}$ and $753 \mathrm{~K}\left(\varphi=80^{\circ}, \psi=15^{\circ}\right)$. Comparison of calculated (circles) and experimental Bragg reflections of domains based on all possible domain states in the trigonal phase: (a) positions are calculated using the orientation matrix of the domain TR1; (b) positions are calculated using the orientation matrix of the domain TR3.

${ }^{\mathrm{TR} 3} \boldsymbol{M}_{\boldsymbol{t}}$ of all possible domain states according to Eq. (9) [23] with respect to the orientation matrix $\boldsymbol{M}_{3}$ of domain TR3. We calculated the orientation matrixes of the domains taking domain TR3 as the "reference" now. Positions of reflections are given in Fig. 8b. The figure confirms that domain TR3 is connected with domain TR1 by a (121) mirror plane and with domain TR4 by the plane (110). However, there is no stress-free wall between TR3 and TR2 domains. It should be noted that indices of the (101) Bragg reflection from domain TR4 obtained by indexing the Laue diffraction pattern can be also determined through cyclic permutation of $(01 \overline{1})$, which was obtained taking domain TR3 as "reference".

Based on the identification of domain walls between the four observed domain states we can assume that the LSGM05 crystal has a "chevron-like" domain configuration in the trigonal phase (Fig. 5d in [23]). In Refs. $[23,24]$ we demonstrated that such structure allows four different orientation states to coexist without additional stress in the trigonal phase. The wall (110) extends the domain wall (011), which connects domains TR1 and TR2. Domain TR3 is connected with the domain TR1 through the domain wall (121), which extends the domain wall (121) between the states TR2 and TR4 and turns at about $90^{\circ}$ at the intersection of the walls (011). Domain walls (121) between domain pairs TR2-TR4 and TR1-TR3 ( $B-C$ and $M-A$ pairs in Fig. 5d [23]) correspond to mirror reflection with respect to domain walls (011) or (110), correspondingly. All four domain states TR contact each other in the direction $[100]_{\mathrm{p}}$ (Fig. $5 \mathrm{~d}$ in [23]).

\subsection{Orthorhombic phase}

In the same sample, a "chevron-like" twin structure was observed in the orthorhombic phase at room temperature. Figure 9 shows a corresponding part of the Laue diffraction pattern observed for sample-to-detector distance of $358 \mathrm{~mm}$. Using the transformation matrices $\boldsymbol{T}_{1 t}$ (see Table 2 in [23]) and the orientation matrix $\boldsymbol{M}_{3}$ (Sect. 4.1) of the "reference" domain state TO3, the reflection positions for domain states related by possible 
twin laws [23] were calculated. In addition to reflections from four TO domains, the calculated reflection positions with respect to the orientation state TO3 ("reference" domain) from possible domain states in the orthorhombic phase are shown in Fig. 9. As seen in the figure, calculated reflection positions from possible domain states connected with domain state TO3 by mirror planes (101) and (121) plane coincide with the positions of the observed (121) and (200) reflections from domain states TO1 and TO2, respectively. The same Miller indices for both reflections were determined using the corresponding transformation matrices $\boldsymbol{T}_{1 t}$ (Table 2 in [23]) and orientation matrix $\boldsymbol{M}_{3}$ of the "reference" domain TO3.

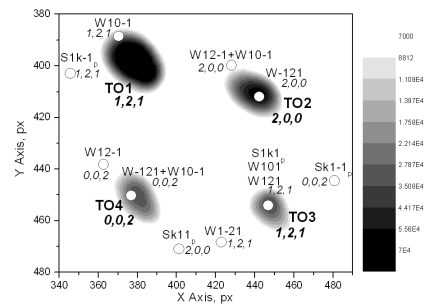

Fig. 9. Section of Laue diffraction pattern detected at $358 \mathrm{~mm}$ and room temperature $\left(\varphi=80^{\circ}, \psi=15^{\circ}\right)$. Comparison of calculated (circles) and experimental Bragg reflections of domains based on all possible domain states in the orthorhombic phase. Positions are calculated with respect to the orientation matrix of domain TO3.

The position of the reflection (002) from domain related with TO3 by the mirror plane $(12 \overline{1})$ rather poorly agrees with the position of the observed reflection from the TO4 state (Fig. 9).

Since, according to [23] 4-domain stress-free junctions

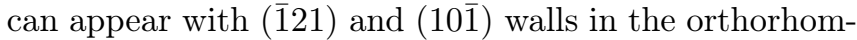
bic phase (see Fig. 10d in [23]), we also calculated the reflection positions for domain state related with domain state TO3 by two subsequent symmetry operations, the mirror planes $(10 \overline{1})$ and $(12 \overline{1})$ as well as the mirror planes $(10 \overline{1})$ and $(\overline{1} 21)$. The reflection position from the domain connected with TO3 via two subsequent mirror reflections with respect to $(\overline{1} 21)$ and (10 $\overline{1})$ is represented as the sum $W(-121)+W(10-1)$ in Fig. 9. The reflection position of $W(-121)+W(10-1)$ agrees better with experimentally observed reflection from state TO4. Thus, we conclude that the domain TO3 is related with TO1

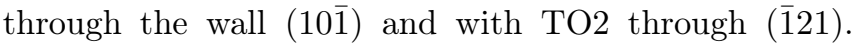
However, the domain state TO4 is not related with TO3,

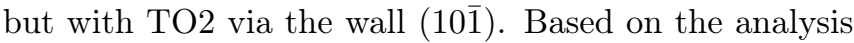
above we find the twin structure shown in Fig. 10d in [23]. The crystallographic plane $(\overline{1} 21)$ is the contact face between domains TO3 and TO2 (domains $A$ and $C$ in Fig. 10d [23], correspondingly), while domains TO1 and TO4 (domains $M$ and $B$ in Fig. 10d [23], correspond-

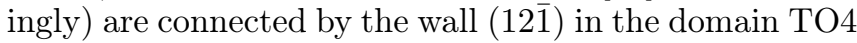
setting. It extends the wall (121) between domains TO3 and TO2. The wall (101) connects the TO3 and TO1 domains as well as TO2 and TO4. This wall changes its orientation at ca. $90^{\circ}$ at intersection with the wall (12) or (121) (Fig. 10d in [23]). Similar to the trigonal phase, the perovskite direction $[100]_{\mathrm{p}}$ is common for all four TO domains. This configuration allows junctions of four different orientation states without additional stress over the temperature range of the orthorhombic phase [24].

\section{Configuration reversibility of twin walls}

The Laue method can be used to investigate the twin structure in different phases as well as its rearrangement at the phase transition. Using this method, the configuration reversibility of twinning walls in LSGM05 during heating above the transition point was studied. The multiplet consisting of reflections (121) from TO1 and TO3, as well as (200) from TO2 and (002) from TO4 and their corresponding reflections of higher order were selected to study temperature-induced changes of the twin pattern. This multiplet is the most intensive in both, the orthorhombic and trigonal phases and allows us to record these reflections during exposure time of $0.1 \mathrm{~s}$.

The crystal was slowly heated with the rate $400 \mathrm{~K} / \mathrm{h}$ up to $900 \mathrm{~K}$. During heating the Laue patterns were recorded. Parallel shift of all four X-ray spots due to thermal expansion and corresponding lattice parameters changes were observed in the orthorhombic phase and above $570 \mathrm{~K}$ in the monoclinic phase. Obviously, the phase transition of the second type from orthorhombic to monoclinic symmetry does not lead to a rearrangement of the ferroelastic domain structure, because all domain walls, which can appear in the orthorhombic phase, can connect domain pairs in the monoclinic low-symmetry phase (Tables 2 and 3 in [23]).

Fundamental changes in spacial distribution of reflections was observed above $670 \mathrm{~K}$ (Fig. 10). Areas of the Laue patterns, shown in Fig. 10b-h, were obtained after $1 \mathrm{~s}$ exposure time, which demonstrate the evolution of the rearrangement of the domain structure during continuous heating of sample. Firstly, the most intensive "old" reflections split into two close peaks (Fig. 10b). Then three no clearly pronounced additional reflections appeared with increasing intensities, whereas the intensities of four "former" peaks decreased (Fig. 10c-g). The "former" low-temperature reflections disappeared completely on further heating (Fig. 10h). It should be noted that intensive $\mathrm{X}$-ray scattering was observed in the areas between coexisting "former" and "new" peaks with a parallelogram-shaped intensity distribution (Fig. 10c-g). The X-ray intensities (Fig. 10h) corresponded to orientation states of the trigonal phase and were identified as reflections (1-10) of state TR1, (211) of TR2 and TR3, (-101) of TR4 in Sect. 4.1 at $753 \mathrm{~K}$. The presence of 8 reflections (Fig. 10c) from both monoclinic and trigonal phases testifies the coexistence of two phases. The sample area switched to the trigonal phase is already divided into four domain states forming a chevron-like configuration of the ferroelastic domain structure. 


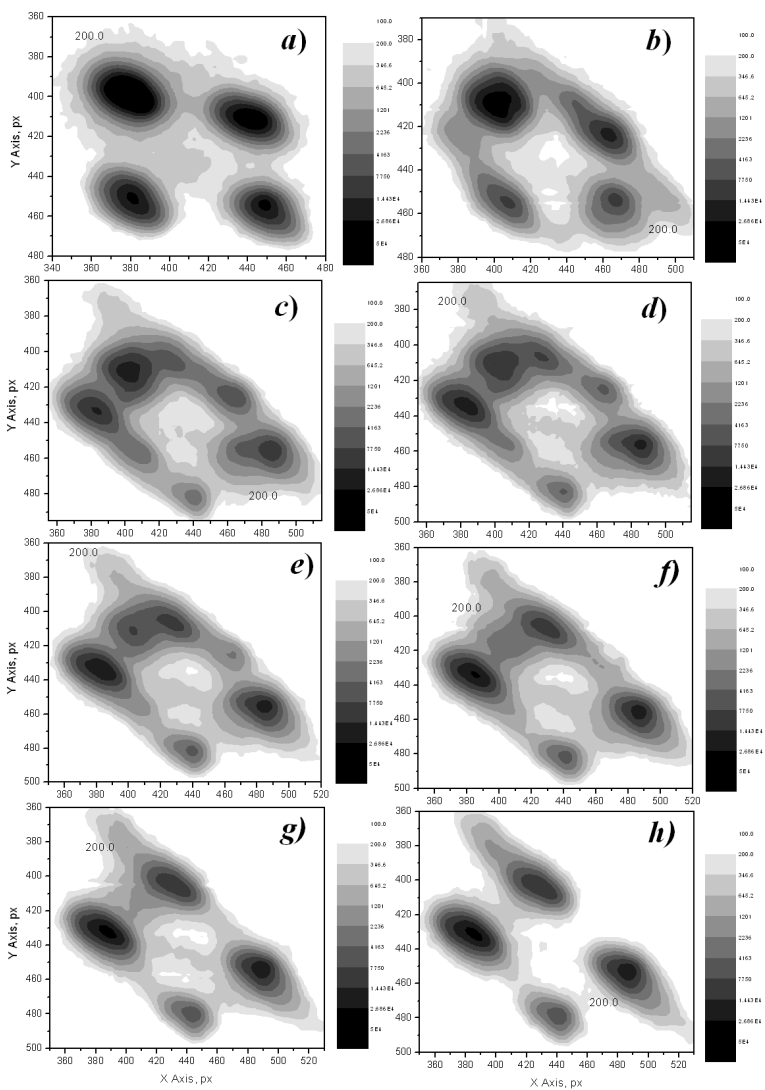

Fig. 10. Enlarged section of the Laue diffraction pattern detected during heating the LSGM05 crystal from 300 (a) to $753 \mathrm{~K}(\mathrm{~h})$. Intensity changes of the Bragg reflections were observed at $670 \mathrm{~K}(\mathrm{~b})-(\mathrm{g})$.

In the low temperature orthorhombic and monoclinic phase the ferroelastic domain structure (Sect. 5.2) rearranges and forms another domain structure (Sect. 5.1) related to the trigonal ferroelastic phase above phase transition (ca. $670 \mathrm{~K}$ ). The configuration of domain walls rearranges again during further cooling at approximately $665 \mathrm{~K}$ (Fig. 11). On cooling we observed the coexistence of four domains of the trigonal phase as well as again the appearance of four domains of the monoclinic phase (Fig. 11b-d), similar to the domain coexistence during heating (Fig. 10). It should be noted that the orientation of domain walls is the same in both the ferroelastic monoclinic (or the orthorhombic) and the trigonal phases with respect to their orientation in the perovskites setting (Fig. 10 and Fig. 5 in [23]). At the phase transition on heating as well as on cooling only some domain walls are rearranged and produced "chevrons rotation" on approximately $45^{\circ}$ in the plane normal to the domain walls.

Figure 12 shows the Laue diffraction patterns (the same multiplet as in Sect. 5.2) before and after heating the crystal above the trigonal transition point for the first three temperature cycles. Since, the crystal was heated above $720 \mathrm{~K}$, the twin structure of the low temperature orthorhombic phase was re-arranged. Upon cooling to
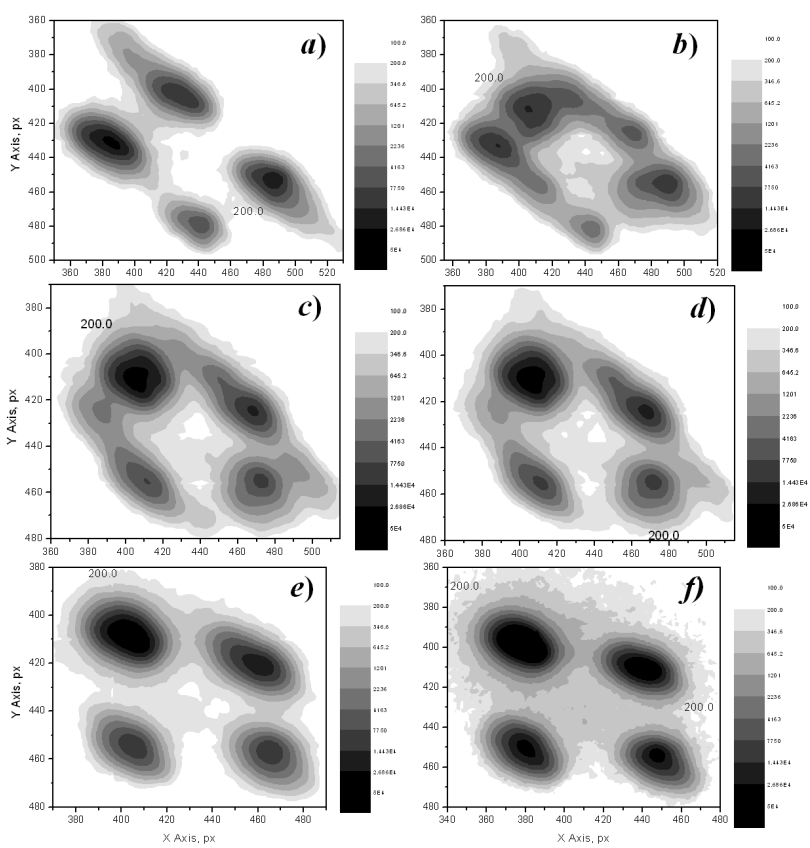

Fig. 11. Enlarged section of the Laue diffraction pattern detected during cooling the LSGM05 crystal from 753 (a) to $300 \mathrm{~K}$ (f). Intensity changes of Bragg reflections were observed at $665 \mathrm{~K}(\mathrm{~b})-(\mathrm{e})$.

room temperature the twin walls rearrange again. One might have expected that this cycling should change the initial configuration of twin walls in the orthorhombic phase. However (see Fig. 12) such change happens only during the first heating cycle, while all Laue diffraction patterns observed in the next cycles are practically identical. After the first heating cycle (see Fig. 12b) the crystal maintains its initial orientation states (Fig. 12a), however, the change of the intensities of the reflections corresponding to the four states indicate changes of the domain volumes. Also the shape of the reflections changes from circular (before heating) to elliptical after the first heating cycle. The Laue diffraction patterns determined after the 2nd and 3rd thermal cycles are practically identical to the Laue diffraction pattern observed after the initial heating cycle. Theefore, the subsequent two thermal cycles have no considerable influence on the configuration of the twin structure in the orthorhombic phase. Reflection positions, shapes and intensities remain practically unchanged after the first thermal treatment.

Insignificant changes of reflections intensities may be explained by the approximation error of the reflections on corresponding Laue patterns. To test this, we determined and analyzed intensities and FWHM data of 12 different multiplets observed below the phase transition for a sample-detector distance of $108 \mathrm{~mm}$ during the 2 nd cycle. This distance was selected as an optimum because it allowed us to separate reflections from different orientation states, and about 20 multiplets were detected using this CDD-detector distance simultane- 


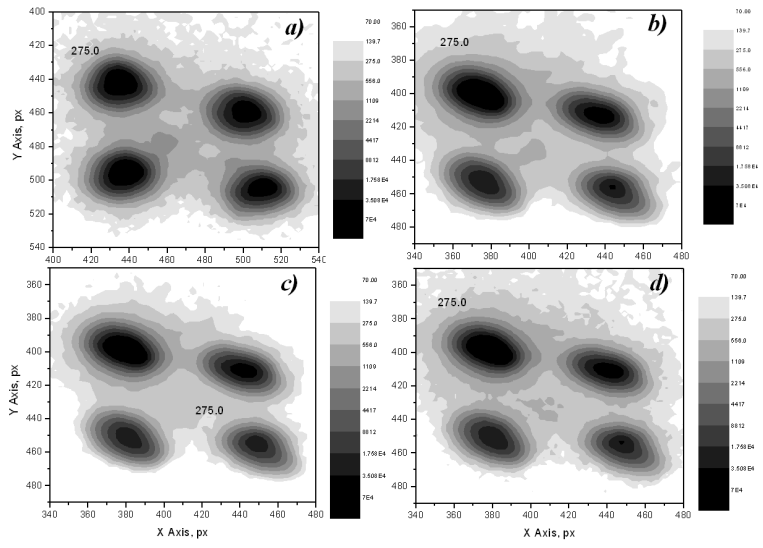

Fig. 12. Sections of the Laue diffraction patterns of the LSGM05 crystal observed at $300 \mathrm{~K}$ before 1st (a) and after the 1st (b), before 3rd (c) and after the 3rd (d) heating cycle for $358 \mathrm{~mm}$ CCD-sample distance.

ously. Intensity and FWHM ratios of all four domains (TO1-TO4) at room temperature before $\left(I_{C 2}, W_{C 2}\right)$ and $\left(I_{C 3}, W_{C 3}\right)$ the 2 nd thermal cycle.

\begin{tabular}{|c|c|c|c|c|c|c|c|c|c|c|c|}
\hline \multicolumn{3}{|c|}{ TO1 } & \multicolumn{3}{|c|}{ TO2 } & \multicolumn{3}{|c|}{ TO3 } & \multicolumn{3}{|c|}{ TO4 } \\
\hline$h k l$ & $I_{\mathrm{C} 2} / I_{\mathrm{C} 3}$ & $W_{\mathrm{C} 2} / W_{\mathrm{C} 3}$ & $h k l$ & $I_{\mathrm{C} 2} / I_{\mathrm{C} 3}$ & $W_{\mathrm{C} 2} / W_{\mathrm{C} 3}$ & $h k l$ & $I_{\mathrm{C} 2} / I_{\mathrm{C} 3}$ & $W_{\mathrm{C} 2} / W_{\mathrm{C} 3}$ & $h k l$ & $I_{\mathrm{C} 2} / I_{\mathrm{C} 3}$ & $W_{\mathrm{C} 2} / W_{\mathrm{C} 3}$ \\
\hline 343 & 0.9388 & 1.047 & 501 & 0.9901 & 0.9871 & $3-43$ & 0.9881 & 1.029 & 105 & 1.028 & 0.9737 \\
\hline 231 & 1.061 & 0.971 & 310 & 1.044 & 0.9571 & $1-32$ & 0.9672 & 1.024 & $0-13$ & 1.029 & 0.9683 \\
\hline 7105 & 0.9341 & 1.054 & 1121 & 1.029 & 0.9967 & $5-107$ & 1.022 & 0.9855 & $1-211$ & 1.034 & 0.9904 \\
\hline 463 & 1.056 & 0.9935 & 1321 & 1.021 & 0.9713 & $3-64$ & 0.9867 & 1.002 & $1-213$ & 1.016 & 0.9934 \\
\hline 9147 & 1.009 & 1.002 & 1521 & 1.053 & 0.9728 & $7-149$ & 0.9602 & 1.053 & $1-215$ & 0.9436 & 1.046 \\
\hline 7107 & 1.01 & 1.008 & $\begin{array}{lll}6 & 0 & 1\end{array}$ & 0.9944 & 1.001 & $7-107$ & 1.02 & 0.9761 & 106 & 1.001 & 1.021 \\
\hline 232 & 1.047 & 0.9907 & $\begin{array}{llll}7 & 0 & 1\end{array}$ & 1 & 0.9931 & $2-32$ & 0.9411 & 1.085 & 107 & 1.013 & 1.007 \\
\hline 364 & 1.052 & 0.9843 & $13-21$ & 0.9639 & 1.036 & $4-63$ & 0.9891 & 0.9928 & 1213 & 0.9888 & 0.9924 \\
\hline 7149 & 0.9789 & 1.015 & $15-21$ & 0.9665 & 1.011 & $9-147$ & 1.216 & 0.8132 & 1215 & 0.9237 & 1.096 \\
\hline 9145 & 1.034 & 0.9848 & 720 & 0.9655 & 1.059 & $5-149$ & 0.9484 & 1.06 & $0-27$ & 1.033 & 0.9946 \\
\hline 583 & 0.9667 & 1.03 & 410 & 0.9421 & 1.067 & $3-85$ & 0.9484 & 1.041 & $0-14$ & 1.138 & 0.8978 \\
\hline 7145 & 1.088 & 0.9579 & $132-1$ & 1.01 & 1.04 & $5-147$ & 1.111 & 0.9374 & $-1-213$ & 0.975 & 1.057 \\
\hline
\end{tabular}

Intensity, FWHM ratios of reflections of 4 TR domain states in the trigonal

TABLE VI after $\left(I_{C 3}, W_{C 3}\right)$ the 2 nd cycle are shown in Table V. Reflections with medium intensity values are selected for comparison. The analysis shows that even small deviations of intensity ratios from some constants involve variations of FWHM ratios in opposite directions. This underlines that deviations in the intensity ratios are caused by the approximation error of reflections.

Reversibility of the twin walls configuration was also observed in the high temperature rhombohedral phase as indicated by identical Laue diffraction patterns, which were detected above the ferroelastic phase transition point. Positions, shapes and intensities of reflections (-110) from TR1, (211) from TR2 and TR3, and (-101) from TR4 are the same after the first and further heat treatments (Fig. 13). Table VI contains the intensity and FWHM values of the above mentioned reflections of 4 domains (TR1-TR4) determined at $690-710 \mathrm{~K}$ in the trigonal phase at successive thermal cycles. For illustration we include the $I_{\mathrm{TR} i} / I_{\mathrm{TR} 1}$ value determined versus the intensity of the reflections $(-110)$ (and its higher orders) from the state TR1. Similarly to the orthorhombic phase, there is a slight difference in values determined at different thermal cycles. This is mainly caused by the error of the approximation of the Laue diffraction spots.
TABLE V

Reflection intensity $(I)$ and FWHM ratios of 4 TO domains at $300 \mathrm{~K}$ before $\left(I_{C 2}, W_{C 2}\right)$ and after

\begin{tabular}{c|c|c|c|c|c|c|c|c}
\hline \hline Domain & \multicolumn{2}{|c|}{ TR1 } & \multicolumn{2}{c|}{ TR2 } & \multicolumn{2}{c|}{ TR3 } & \multicolumn{2}{c}{ TR4 } \\
\hline Reflection indices & \multicolumn{2}{|c|}{-110} & \multicolumn{2}{c|}{211} & \multicolumn{2}{c|}{211} & \multicolumn{2}{c}{-101} \\
\hline $\begin{array}{c}\text { Cycle and sample- } \\
\text { CCD distance }\end{array}$ & $I, I / I_{\mathrm{TR} 1}$ & $W$ & $I, I / I_{\mathrm{TR} 1}$ & $W$ & $I, I / I_{\mathrm{TR} 1}$ & $W$ & $I, I / I_{\mathrm{TR} 1}$ & $W$ \\
\hline 1st, $358 \mathrm{~mm}$ & $25183, \mathbf{1}$ & 16.965 & $11002, \mathbf{0 . 4 4}$ & 17.764 & $21004, \mathbf{0 . 8 3}$ & 14.523 & $5939, \mathbf{0 . 2 4}$ & 14.454 \\
2nd, $108 \mathrm{~mm}$ & $64460, \mathbf{1}$ & 8.547 & $31333, \mathbf{0 . 4 9}$ & 9.060 & $47435, \mathbf{0 . 7 4}$ & 7.317 & $13527, \mathbf{0 . 2 1}$ & 8.416 \\
3rd, 358 mm & $29772, \mathbf{1}$ & 16.649 & $12304, \mathbf{0 . 4 1}$ & 18.544 & $22457, \mathbf{0 . 7 5}$ & 14.638 & $6336, \mathbf{0 . 2 1}$ & 14.667 \\
5 th, $58 \mathrm{~mm}$ & $22048, \mathbf{1}$ & 6.105 & $9489, \mathbf{0 . 4 3}$ & 8.126 & $15403, \mathbf{0 . 7 0}$ & 6.287 & $3962, \mathbf{0 . 1 8}$ & 8.798
\end{tabular}

Our results show that orientation states and domain walls between them are reproduced without noticeable changes in both, the low temperature orthorhombic and the high temperature trigonal phase during the ferroelas- 
tic transition. Investigation of the multiplet intensity in the Laue diffraction patterns collected at different temperatures showed that the successive twin configurations appearing during the temperature cycling between orthorhombic and trigonal phases can be described as follows:

$$
Z \rightarrow X \rightarrow Y \rightarrow X \rightarrow Y \rightarrow \ldots
$$

where $Z$ is the initial twin configuration in the orthorhombic phase prior to the first heating above the trigonal transition point, $X$ is the twin configuration formed in the trigonal phase after heating (Sect. 5.1), $Y$ is the twin configuration appearing in the orthorhombic phase (instead of the initial one) after cooling below the trigonal transition point (Sect. 5.2). Thus, after the first thermal cycle the crystal can "switch" between two phase-specific twin configurations. Additionally, the volume of each orientation state is reproduced.
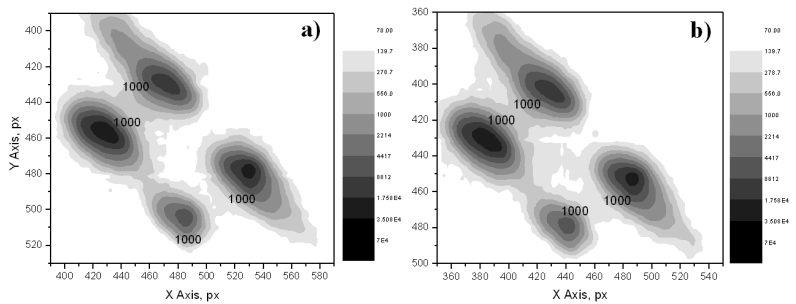

Fig. 13. Section of the Laue diffraction patterns of LSGM05 observed on heating in the trigonal phase at the 1st $(710 \mathrm{~K})(\mathrm{a})$ and 3rd $(690 \mathrm{~K})$ (b) thermal cycles with $358 \mathrm{~mm}$ CCD-sample distance.

This is a full reproducibility of the domain walls configuration in the sample during thermal cycles. In other words, there is a domain wall location memory effect. Only the twin structure observed in the orthorhombic phase prior to the first phase transition is an exception. In order to understand this behavior it should be taken into account that the sample was prepared through mechanical fragmentation at room temperature, which causes additional strain in the crystal. Stress in LSGM05 crystals can easily relax via the separation into different orientation states. The additional induced stress results in a rearrangement of the twin structure, which was listed before the sample preparation. The corresponding twin structure is a "non-equilibrium" state because both, the crystal shape and its size changed after mechanical fragmentation. The "equilibrium" twin structure is then formed in the trigonal phase on heating above the transition point. This structure occurs due to the relaxation of stress caused by the mismatch of neighboring phases at the phase front and crystal defects under new boundary conditions, involving the crystal size and shape. Subsequent cooling of the sample below the phase transition point results in the rearrangement of the walls according to the distribution of stress in the low temperature phase induced by the same defects and mismatch of phases at the phase front. Reproducibility of the twin configura- tions in both phases indicates reproducibility of the stress distribution. Since the stress fields are formed by two factors, i.e. mismatch of the phases and defects, the reproducibility of the configuration of the twin structure results from the reproducibility of stress fields induced by those two factors or one of them.

Namely, domination of mechanical strain due to mismatch of two phases results in a periodical distribution of strain [27]. It is difficult to expect the reversibility of the strain from one temperature cycle to the next one, because it is defined by external conditions, i.e. by the temperature field. The smallest fluctuations in temperature cause a change of the phase front orientation and a different strain distribution, which results in a different periodical size and configuration of the ferroelastic domain structure.

Defects create an irregular stress distribution due to their arbitrary localization in the sample. According to [28-32] they maintain their distribution in the crystal and determine the reversibility of strain fields because of their insignificant migration at comparatively low temperatures $(\approx 700 \mathrm{~K})$. At the transition to the paraelastic phase (or ferroelastic phase as in our case) the defect distribution remains unchanged and the same configuration of domains walls on cooling to the low temperature ferroelastic phase occurs [33]. In case of LSGM05 a phase transition from one ferroelastic phase to another one takes place, but the mechanism of reversibility of domain wall configurations is the same. Furthermore, a "chevron-like" configuration appears in the high-temperature ferroelastic trigonal phase, where the domain walls are parallel to the domain walls of the low-temperature ferroelastic orthorhombic phase (Sect. 5). Such topological correlation of domain wall configurations in both phases is not accidental and testifies about dominating influence of point defects on the configuration of the domain pattern in LSGM05. We may expect an accumulation of bivalent cations $\left(\mathrm{Sr}^{2+}, \mathrm{Mg}^{2+}\right)$ and oxygen vacancies inside or close to the domain walls.

\section{Conclusions}

Our results show that heating from the orthorhombic to the trigonal phase and cooling from the trigonal phase to the orthorhombic one causes "chevron-like" twin patterns in LSGM05 crystals. All "chevron cells" are extended along the same perovskite axis $\langle 001\rangle_{\mathrm{p}}$, in both, the orthorhombic and the trigonal phase. Besides, four orientation states occur which perfectly match geometrically and hence no additional stress occurs at the intersections of the domain walls throughout the full stability range of the corresponding phases.

The specific "chevron" twin pattern allows one the reproducibility of wall configurations in heavy defect LSGM05 crystals. The stress can completely relax by forming phase-specific domain wall configurations, and hence, reorientations occur during thermal cycling. Such pattern of domain walls is characteristic also for other 
perovskite-type compounds with a sequence of ferroelastic phase transitions related to those of LSGM05. Examples are mixed conductivity perovskites, which are used as electrode materials and interconnectors in SOFC batteries.

The reversibility of characteristic chevron-like domain patterns during the transformation between ferroelastic phases of LSGM05 crystal was studied in detail. The observed reversibility of the domain structure in this crystal follows from the distribution of microstrain caused by the spatial distribution of oxygen vacancies and dopant ions in the crystal. This distribution remains stable because the migration of vacancies and ions in the crystal structure at temperatures about $750 \mathrm{~K}$ is small. Taking into account the segregation of oxygen vacancies at domain walls, it has been assumed that the high ionic conductivity of LSGM compounds is caused by two simultaneous processes of oxygen diffusion: through the volume of the domains and fast along the domain boundaries $[7,8]$.

In the trigonal and orthorhombic phase a "chevron-like" configuration is formed due to atomic displacements along the direction $\langle 001\rangle_{\mathrm{p}}$, parallel to the "chevron cells". This feature may be of practical use because the preparation of electrolyte and electrode ceramics for SOFC includes compaction as one of the synthesis stages. Compaction leads to unidirectional mechanical stress. Ceramics can therefore be approximated by an ensemble of small crystallites and mechanical pressure imposed to an electrolyte pellet, which causes rearrangement of the twin structure of "chevron cells" in ceramic grains along the direction parallel or nearly parallel to the imposed pressure. Hence, such pressure will cause texturing of twin "chevrons" in electrolyte layers along the direction of oxygen diffusion in the SOFC structure. Keeping in mind the influence of twin walls on the conductivity and the high density of twin walls in LSGM05 [7, 8], we suppose that texturing of the twin structure, e.g. reorientation of "chevron cells" increases the conductivity of the perovskite-type electrolyte LSGM in the cathode-anode direction.

\section{Acknowledgments}

We thank S. Heidrich for microprobe analysis. The work was supported by WTZ (UKR 07/009) and Ukrainian Ministry of Education and Science (the "Tern" project).

\section{References}

[1] B.C.H. Steele, A. Heinzel, Nature 414, 345 (2001).

[2] R.N. Vannier, S.J. Skinner, R.J. Chater, Solid State Ionics 160, 85 (2003).

[3] I. Abrahams, F. Krok, J. Mater. Chem. 12, 3351 (2002).

[4] F.M. Morales, M. Ruhle, Acta Crystallogr. B 62, 761 (2006).

[5] T. Mori, J. Drennan, J.-H. Lee, Solid State Ionics 154-155, 461 (2002).
[6] A. Skowron, P. Huang, A. Petric, J. Solid State Chem. 143, 202 (1999).

[7] M. Kurumada, E. Iguchi, D. Savytskii, J. Appl. Phys. 100, 014107 (2006).

[8] E. Iguchi, D. Savytskii, M. Kurumada, in: Diffusion and Reactivity of Solids, Eds. Ja.Y. Murdoch, Nova Sci. Publ., New York 2007, p. 115.

[9] Ju.L. Flippen-Anderson, Je.R. Deschamps, R.D. Gilardi, Cryst. Eng. 4, 131 (2001).

[10] R.F. Klie, Y. Ito, S. Stemmer, Ultramicroscopy 86 , 289 (2001).

[11] F. Tsai, V. Khiznichenko, J.M. Cowley, Ultramicroscopy 45, 55 (1992).

[12] C.L. Jia, K. Urban, Science 303, 2001 (2004).

[13] S. Stemmer, A. Sane, N.D. Browning, Solid State Ionics 130, 71 (2000).

[14] Y. Wang, Science 248, 468 (1990).

[15] Y. Wang, Science 251, 410 (1991).

[16] W.L. Wang, H.Y. Lu, Phys. Chem. Minerals 130, 71 (2006).

[17] W.L. Wang, H.Y. Lu, J. Am. Ceram. Soc. 89, 281 (2006).

[18] D. Savytskii, D. Trots, L. Vasylechko, C. Paulmann, U. Bismayer, M. Berkowski, HASYLAB Annual Reports, p. 515 (2003).

[19] D. Savytskii, D. Trots, L. Vasylechko, C. Paulmann, U. Bismayer, M. Berkowski, HASYLAB Annual Reports, p. 513 (2003).

[20] D.I. Savytskii, D.M. Trots, L.O. Vasylechko, N. Tamura, M. Berkowski, J. Appl. Crystallogr. 36, 1197 (2003).

[21] A.A. MacDowell, R.S. Celestre, N. Tamura, Nucl. Instrum. Methods Phys. Res. A 467-468, 936 (2001).

[22] N. Tamura, R.S. Celestre, A.A. MacDowell, Rev. Sci. Instrum. 73, 1369 (2002).

[23] D. Savytskii, T. Tataryn, N. Martynyuk, U. Bismayer, Acta Phys. Pol. A 117, 48 (2010).

[24] D. Savytskii, U. Bismayer, Phase Transit. 81, 431 (2008).

[25] L. Vasylechko, V. Vashook, D. Savytskii, A. Senyshyn, R. Niewa, M. Knapp, H. Ullmann, M. Berkowski, A. Matkovskii, U. Bismayer, J. Solid State Chem. 172, 396 (2003).

[26] S. Riquet, K.J. Bonnet, Appl. Crystallogr. 12, 39 (1979).

[27] A.L. Roytburd, Phase Transit. 45, 1 (1993).

[28] C. Frondel, Am. Mineral. 30, 447 (1945).

[29] P.J. Heaney, D.R. Veblen, Am. Mineral. 76, 1459 (1991).

[30] H.W. Xu, P.J. Heaney, Am. Mineral. 82, 99 (1997).

[31] S.A. Hayward, E.K.H. Salje, Mineral. Mag. 64, 195 (2000).

[32] F. Camara, J.C. Doukhan, E.K.H. Salje, Phase Transit. 71, 227 (2000).

[33] E.K.H. Salje, S.A. Hayward, W.T. Lee, Acta Crystallogr. A 61, 3 (2005). 